\title{
Poor adhesion of fentanyl transdermal patches may mimic end-of-dosage failure after 48 hours and prompt early patch replacement in hospitalized cancer pain patients
}

\author{
This article was published in the following Dove Press journal: \\ Journal of Pain Research \\ 9 November 2016 \\ Number of times this article has been viewed
}

\section{Isabelle Arnet' \\ Sabrina Schacher' \\ Eva Balmer ${ }^{2}$ \\ Dieter Koeberle ${ }^{2}$ \\ Kurt E Hersberger ${ }^{\prime}$ \\ 'Department of Pharmaceutical Sciences, Pharmaceutical Care Research Group, University of Basel, ${ }^{2}$ Palliative Care Unit, Division of Oncology/Haematology, St Claraspital, Basel, Switzerland}

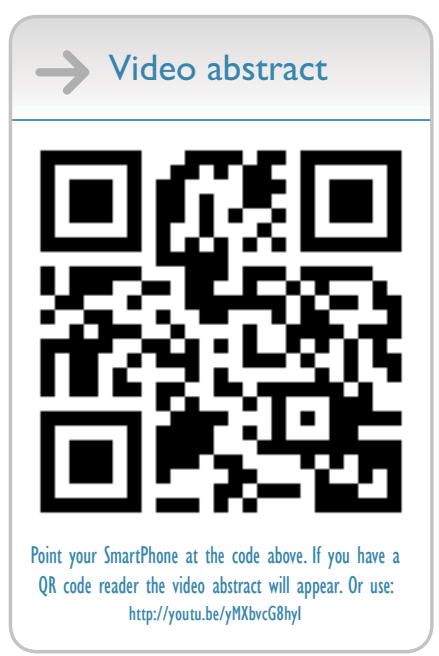

Correspondence: Isabelle Arnet Department of Pharmaceutical Sciences, Pharmaceutical Care Research Group, University of Basel, Klingelbergstrasse 50, 4054 Basel, Switzerland

Tel +4I 6I $267 \mid 567$

Fax +4I 6I 267 I428

Email isabelle.arnet@unibas.ch

\begin{abstract}
Context: Renewal of fentanyl transdermal patch (transdermal therapeutic system [TTS]) should occur every 3 days (72 hours) according to manufacturer's guidelines. Some studies mentioned patients reporting end-of-dose failure, and thus, some authors recommend shortening the interval of application to 2 days ( 48 hours). However, reasons for early replacement are mostly unknown. Objectives: The objectives of this study were to assess the prevalence of early replacement of fentanyl TTS in a cancer center in Basel, Switzerland, and to assess the reasons for early replacement in stationary patients.
\end{abstract}

Methods: We retrieved all fentanyl TTS administered in a cancer center in Basel, Switzerland, between November 11, 2011, and January 31, 2015, from the electronic medical database.

Results: A total of 739 patients (mean age $71.4 \pm 11.5$ years, $55 \%$ women) were administered 2,250 fentanyl TTS (dosage 6-500 $\mu \mathrm{g}$ /hour). Most replacements occurred after 72 hours (61.6\%) and a few after 48 hours (7.4\%). Patients with early replacement after 48 hours were significantly younger ( 63.8 years versus 71.5 years, $p<0.001$ ) and obtained higher mean dosages of fentanyl TTS ( $89 \mu \mathrm{g} / \mathrm{hour}$ versus $44.1 \mu \mathrm{g} / \mathrm{hour}, p<0.001$ ) and rescue medication (calculated as oral morphine equivalent in milligrams: $185.1 \mathrm{mg}$ versus $39.6 \mathrm{mg}$ during the first 24 hours after replacement, $p<0.001$ ). No pharmacological rationale for early replacement was observed. According to 57 physicians, nurses, and pharmacists, the most often reasons for early TTS replacement were end-of-dosage pain (41.4\%) and poor adhesion (31.4\%).

Conclusion: In the absence of any physiological, pharmacological, or environmental reasons recorded in the database to explain an early replacement of fentanyl TTS, skin adhesion problems may point practical reasons and mimic end-of-dosage failure.

Keywords: transdermal fentanyl, long-term therapy, early replacement, dosing frequency, chronic pain

\section{Introduction}

Fentanyl is a strong opioid agent, which is $\sim 100$ times more potent than morphine. ${ }^{1}$ It is commercially available as a patch or transdermal therapeutic system (TTS) that delivers drug to the skin surface and provides constant drug delivery to the general circulation for several days. Fentanyl TTS represents a cornerstone in the treatment of patients with severe pain and is convenient for patients with continuous severe pain in need of a drug with stable pharmacokinetic profiles. The renewal of the patch should occur every 3 days ( 72 hours) according to manufacturer's guidelines. A retrospective chart review in a US pain center revealed that nearly every patient 
reported perceived end-of-dosage failure as the reason for taking the medication more frequently. ${ }^{2}$ Consequently, some researchers recommend early replacement of the patches every 48 hours, predominantly if pain increases at the end of the 3-day period and if a higher dosage produces adverse effects without improving pain relief. ${ }^{3}$ More frequent dosing is not recommended by national guidelines or consensus statements, ${ }^{4}$ and no published reference recommends dosing intervals $<48$ hours. Furthermore, the reasons for the early replacement are mostly unknown.

The aims of this study were to assess the prevalence of early replacement of fentanyl TTS in a cancer center in Basel, Switzerland, and to assess the reasons for early replacement in stationary patients.

\section{Methods}

\section{Study design}

This is a retrospective health-related database study.

\section{Setting}

The St Clara hospital is the largest private acute care hospital in Basel, Switzerland, with 229 beds. It gives emphasis on oncology and holds a palliative care unit of six single bedrooms within the cancer center. The entire workflow is digitalized, and electronic patient files are available. The electronic medical database was reviewed for administration of Durogesic ${ }^{\circledR}$ Matrix (fentanyl TTS, all strengths) between November 1, 2011, and January 31, 2015. The extracted data concerned patient (year of birth, gender, ward, main diagnosis, body mass index [BMI]), fentanyl TTS (strength, dosage, application day, and hour according to the confirmation stamp of the dispensing nurse), co-medication (name, strength, and dosage), co-analgesic treatment (name, strength, and dosage, excluding local application forms), laboratory values (aspartate transaminase [AST] and alanine aminotransferase [ALT]), and psychological state (inconspicuous, depressive, agitated, or others). Data from patients with a hospital stay $\geq 10$ days were extracted.

\section{Variables}

The primary measure was the time between two administrations of fentanyl TTS. The secondary measures were the daily use of breakthrough pain medication and of comedications. The total quantity of dispensed breakthrough pain medication over periods of 24 hours was converted into an equivalent dosage of oral morphine (in milligrams) according to conversion tables retrieved from the literature (Supplementary materials).

\section{Bias}

Because patch renewal after 3 days was the topic of interest, patients with a short hospitalization stay, ie, $<10$ days, were excluded.

\section{Survey}

A written survey was developed assessing nurses', physicians', and pharmacists' personal experiences with fentanyl TTS and reasons for early replacement. It combined four openended questions, 23 closed-ended questions (ten $10 \mathrm{~cm}$ visual analog scale [VAS], two yes/no answers, one multiple choice answer) and took 10 minutes to complete. It was distributed during wards' reports and sent back through internal post-mail. The study was approved by the ethics committee Ethikkommission Nordwest- und Zentralschweiz (EKNZ 2015-037). The ethics committee deemed patient consent not necessary.

\section{Quantitative variables}

The values were calculated as mean and standard deviations.

\section{Statistical analysis}

Data depository and statistical analysis were performed with the Statistical Package for the Social Sciences software, version 22 for Windows (IBM Corporation, Armonk, NY, USA). Chi-square and Mann-Whitney $U$ tests were performed when appropriate. The Wilcoxon signed-rank test was used since the data were not normally distributed (Kolmogorov-Smirnov test). The significance level was set at $p<0.05$.

\section{Results}

Of 3,514 patients who were prescribed fentanyl TTS, 1,196 were excluded because of duplicate entry in the database, 1,557 because of hospitalization stay $<10$ days, 11 because of no discharge date, and 11 were ambulatory patients. Data of the remaining 739 patients were analyzed. The median hospital stay was 17 days and mean was 14 days ( \pm 10.7 days) with a maximal length of 110 days (one patient). The patients' mean age was $71.4 \pm 11.5$ years and BMI was $23.8 \pm 4.9 \mathrm{~kg} / \mathrm{m}^{2}$ (values $<25 \mathrm{~kg} / \mathrm{m}^{2}$ were considered normal), and $55 \%$ were women (Table 1). Cancer was the most frequent diagnosis (79.4\%), all in advanced or terminal stages. Pain was the reason for hospitalization of $1.5 \%$ of the patients. Patients' psychological states were mostly not specified (54.8\%) or inconspicuous (38.4\%), and depression was recorded for $2.6 \%$ of the patients. Liver enzymes were normal for $54.5 \%$ of the patients, increased for $37.8 \%$ of them, and not specified for the remaining $7.7 \%$. There was no difference between genders (data not shown). Oral co-medication was 
Table I Characteristics of the 739 hospitalized patients included in the study

\begin{tabular}{lllll}
\hline & Total & Men & Women & p-value \\
\hline Number (\%) & 739 & $336(45.5)$ & $403(54.5)$ & $\mathrm{ns}$ \\
Age, mean years \pm SD & $71.4 \pm 1 \mathrm{I} .5$ & $70.0 \pm 1 \mathrm{I} . \mathrm{I}$ & $72.6 \pm 1 \mathrm{I} .7$ & $\mathrm{~ns}$ \\
(range) & & $(36-96)$ & $(20-101)$ & \\
BMI kg/m², mean \pm SD & $23.8 \pm 4.9$ & $23.8 \pm 3.8$ & $23.9 \pm 5.7$ & $\mathrm{~ns}$ \\
\hline
\end{tabular}

Abbreviations: ns, not significant; SD, standard deviation; BMI, body mass index.

administered to $86.3 \%$ of the patients, mostly in the form of 5 to 10 medications $(50.9 \%)$ or $>10$ medications $(21.2 \%)$.

From the 3,122 fentanyl TTS that were administered, $872(27.9 \%)$ were excluded because of missing information. Of the remaining 2,250 fentanyl TTS with complete data sets, main times of administration were $2 \mathrm{pm}(41.0 \%)$, 8 am (28.3\%), $6 \mathrm{pm}$ (7.1\%) and 12 am (6.7\%). Fentanyl TTS dosages ranged from $6 \mu \mathrm{g} / \mathrm{hour}$ to $500 \mu \mathrm{g} /$ hour with the most often used dosages being $25 \mu \mathrm{g} /$ hour (26.2\%) and $12 \mu \mathrm{g} /$ hour (25.4\%). Replacement intervals ranged from 2 hours to 120 hours, and $61.6 \%$ of the replacements occurred after 72 hours. Replacement after 48 hours was observed for $7.4 \%$ of all administered fentanyl TTS. Patients with early replacement after 48 hours were significantly younger (63.8 years versus 71.5 years; $p<0.001$ ), obtained twofold higher mean dosages of fentanyl TTS $(89.0 \mu \mathrm{g} /$ hour versus $44.1 \mu \mathrm{g} /$ hour; $p<0.001$ ), and had more often cancer (Table 2). Range and significance of the values were maintained after exclusion of the three patients with the highest dosages of fentanyl TTS (>300 $\mu \mathrm{g} /$ hour).

Besides fentanyl, $46 \%$ of the patients obtained co-analgesic agents. Metamizole was the most often used non-opioid co-analgesic, either alone (19.9\%) or in combination with paracetamol (3.8\%) or nonsteroidal anti-inflammatory drugs (2\%). Paracetamol was the second most often prescribed co-analgesic agent (14.4\%). The use of co-analgesic did not differ if the replacement interval for fentanyl TTS was 48 hours or other (data not shown). The CYP 3A4 inducers St John's wort, carbamazepine, and rifampicin were administered to seven patients $(0.9 \%)$ whose fentanyl TTSs were not replaced after 48 hours.

Additional breakthrough pain medication (rescue) was needed by $91.2 \%$ of the patients. Morphine was the most often used rescue medication and was administered as oral drops $(44.2 \%)$, parenteral formulation $(16.9 \%)$, or a combination of both $(22.3 \%)$. For the fentanyl TTSs that were replaced after 48 hours, the mean dosages of breakthrough pain medication (calculated as oral morphine equivalent in milligram) were similarly high during the first day/0-24 hours and the next day/24-48 hours (first day: $185.1 \mathrm{mg}$ versus second day: $157.0 \mathrm{mg}$; Wilcoxon $p=0.57$ ), but 4.5 -fold and 5.5 -fold higher than the fentanyl TTS with other replacement time (first day: $185.1 \mathrm{mg}$ versus $39.6 \mathrm{mg}$; $\mathrm{Chi}^{2}=238.3, p<0.001$; second day: $157.0 \mathrm{mg}$ versus $28.8 \mathrm{mg}$; $\mathrm{Chi}^{2}=227.2, p<0.001$; Table 2). For the fentanyl TTS with other replacement time, the mean dosages of breakthrough pain medication did not change over the three consecutive days (39.6 mg [first day] and $30.2 \mathrm{mg}$ [third day]; Wilcoxon $p=0.83$; Table 2). Range and significance of the values remained after exclusion of the three patients with highest dosages of fentanyl TTS, ie, $>300 \mu \mathrm{g} /$ hour.

We wanted to investigate if the dosages changed from one application to the next, ie, according to the consecutive

Table 2 Characteristics of the 2,250 fentanyl TTS administered and breakthrough (rescue) pain medication (calculated as equivalent dosage of oral morphine in milligrams) for every 24 hours over the first day (at day I), second day (at day 2), and third day (at day 3 ) after replacement of fentanyl TTS

\begin{tabular}{|c|c|c|c|}
\hline & $\begin{array}{l}\text { Early replacement after } 48 \text { hours } \\
(n=166)\end{array}$ & $\begin{array}{l}\text { Other replacement time } \\
(n=2,084)\end{array}$ & p-value \\
\hline Patient mean age, years $\pm S D$ (range) & $63.8 \pm 12.9(36-91)$ & $71.5 \pm 11.0(20-101)$ & $<0.001$ \\
\hline \multicolumn{4}{|l|}{ Patient diagnosis (\%) } \\
\hline Cancer & 94.0 & 82.9 & $<0.001$ \\
\hline Pain & 1.2 & 2.4 & $<0.01$ \\
\hline Pneumonia & 2.4 & 2.5 & ns \\
\hline Fracture/necrosis & 0 & 4.2 & $<0.01$ \\
\hline Cardiovascular & 0 & 2.3 & $<0.01$ \\
\hline Other & 2.4 & 5.1 & $<0.01$ \\
\hline Fentanyl mean dosage, $\mu \mathrm{g} /$ hour $\pm \mathrm{SD}$ (range) & $89.0 \pm 108(6-500)$ & $44.1 \pm 43.9(6-500)$ & $<0.001$ \\
\hline Rescue mean dosage at day I, mg \pm SD (range) & $185.1 \pm 660(0-5,250)$ & $39.6 \pm 179(0-5,250)$ & $<0.001$ \\
\hline Rescue mean dosage at day $2, \mathrm{mg} / 24$ hours $\pm \mathrm{SD}$ (range) & $157.0 \pm 484(0-4,125)$ & $28.7 \pm 62(0-1,080)$ & $<0.001$ \\
\hline Rescue mean dosage at day $3, \mathrm{mg} / 24$ hours $\pm \mathrm{SD}$ (range) & - & $30.2 \pm 58(0-550)$ & - \\
\hline
\end{tabular}

Abbreviations: TTS, transdermal therapeutic system; SD, standard deviation; ns, not significant. 
sequence of the administration of the fentanyl TTS. We hypothesized that dosage between two consecutive administrations of fentanyl TTS would increase for the TTS replaced after 72 hours, as a mirror of the method of choice to obtain pain control. We hypothesized that dosage between two consecutive administrations of fentanyl TTS would remain constant for the TTS replaced earlier after 48 hours, with the rationale that if shortening the application time is the method of choice to obtain pain control, it should not be accompanied by dose increase. After exclusion of the three patients with the highest dosages of fentanyl TTS (>300 $\mu \mathrm{g} /$ hour), we sorted the fentanyl TTS into the first four sequences of administration (first to fourth; $N=1,668$ ). The total number of fentanyl TTS per sequence neither correlated with age (Pearson $r=-0.07$ ) nor with fentanyl dosage (Pearson $r=0.12$ ). From one application to the next, the mean dosages of the fentanyl TTS increased steadily from the first to the fourth application and according to replacement time, and statistical significance was reached between each sequence (Wilcoxon $p<0.001$; Figure 1). For each sequence, the mean dosages of the fentanyl TTS with replacement after 48 hours were significantly higher than the fentanyl TTS with other replacement time (Wilcoxon $p<0.001$; Figure 1).

\section{Questionnaire}

A total of 98 questionnaires were distributed on April 14 and 15,2015 , at the palliative unit, oncology wards, and during physician reports. After 10 days, 57 questionnaires were returned (58\% response). The respondents were in average $37.2 \pm 9.3$ years old, mainly women $(76.8 \%)$, Swiss $(66.1 \%)$ or German $(32.1 \%)$, and nurses $(58.9 \%)$ or physicians $(33.9 \%)$. Half of the respondents were slightly not convinced that the administration of fentanyl TTS every 72 hours would lead to pain relief (a mean value of 7.2 on a VAS from $0=$ not convinced to $10=$ absolutely convinced). In daily practice, the most frequent replacement interval was 72 hours $(96 \%)$ or 48 hours (4\%). For 36 respondents $(63.2 \%)$, early replacement of fentanyl TTS was known from theory and from practice. A total of $27 \%$ of the respondents indicated that $16.7 \%$ of their patients obtained early replacement of fentanyl TTS during the last 30 days. The most frequent reasons evoked were end-of-dosage pain $(41.4 \%)$ and inadequate adhesion owing to inappropriate skin property $(31.4 \%)$. The
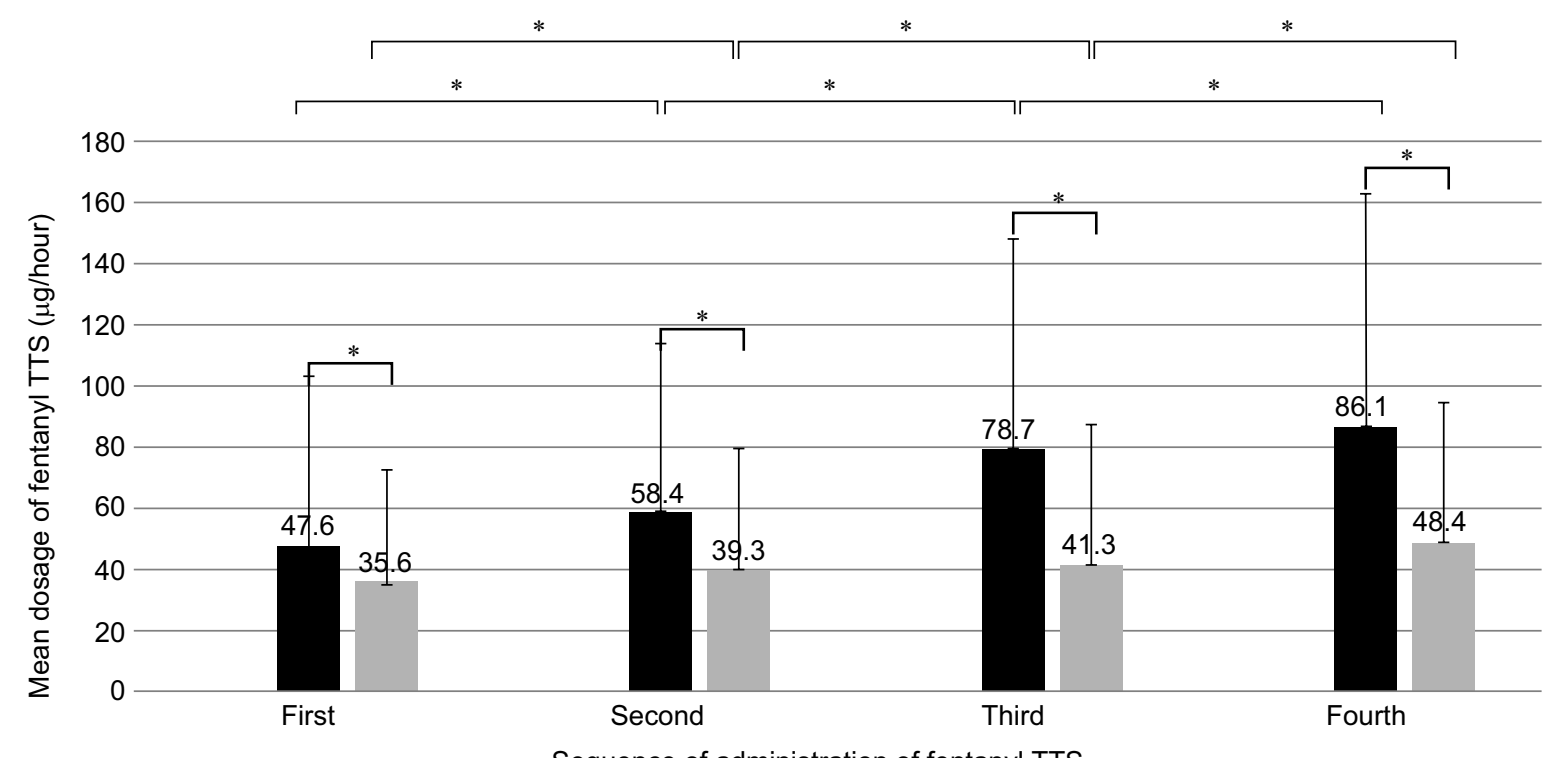

Sequence of administration of fentanyl TTS

\begin{tabular}{|l|l|l|l|l|l|l|l|l|}
\hline $\mathrm{N}=1,668$ & First $(\mathrm{n}=32)$ & First $(\mathrm{n}=582)$ & Second $(\mathrm{n}=29)$ & Second $(\mathrm{n}=439)$ & Third $(\mathrm{n}=23)$ & Third $(\mathrm{n}=330)$ & Fourth $(\mathrm{n}=19)$ & Fourth $(\mathrm{n}=214)$ \\
\hline $\begin{array}{l}\text { Mean } \\
(\mu \mathrm{g} / \text { hour })\end{array}$ & 47.6 & 35.6 & 58.4 & 39.3 & 78.7 & 41.3 & 86.1 \\
\hline $\mathrm{SD}$ & 55.5 & 36.6 & 55 & 36.3 & 69.5 & 36.7 & 76.6 \\
\hline (Range) & $(6-250)$ & $(6-300)$ & $(12-250)$ & $(6-250)$ & $(12-250)$ & $(6-300)$ & $(12-250)$ & $(6-225)$ \\
\hline
\end{tabular}

Figure I The mean dosages of the fentanyl TTS according to the first four sequences of administration (first to fourth) and values in the data table ( $\mathrm{N}=\mathrm{I}, 668)$. Notes: Black column: fentanyl TTS with early replacement after 48 hours $(n=103)$; gray column: fentanyl TTS with other replacement time ( $n=1,565)$. The bars indicate positive SD; ${ }^{*} p<0.001$.

Abbreviations: TTS, transdermal therapeutic system; SD, standard deviation. 
answers did not differ between the different professions. When asked to rate how often the participants encountered different reasons for an early replacement of fentanyl TTS in practice (from $0 \%=$ never to $100 \%=$ always) from a list of eight reasons retrieved from literature, the most frequent reasons were an insufficient dosage of fentanyl (47\%); a dry, sweating, or cachexic skin (36\%); end-of-dosage pain (30\%); and poor adhesion of the TTS (30\%). Drug interaction with CYP 3A4-inducers was never mentioned.

\section{Discussion}

In this cohort of mainly cancer patients, replacement of fentanyl TTS earlier than the prescribed 72 hours was observed in a higher than expected proportion (31\%). However, most of these divergent dosing intervals had been annotated by the nurses. They were related to dosage-finding strategies or inadequate skin adhesion or were the results of a time correction after an erroneous application such as in evening instead of morning. An application period of 60 hours was not used, probably because it would result in an inconvenient change every 2.5 days, such as one change in the morning and the next in the evening. This might also lead to more confusions than every third day.

In our study, the frequency of an application period of 2 days (48 hours) was $7.4 \%$, which was in the range of other studies. ${ }^{5,6}$ Higher opioid dosage and younger age of our patients were associated with early fentanyl TTS replacement. To our knowledge, a similar relationship is mentioned in a Korean study performed in cancer patients taking sustainedrelease opioids - oral morphine, oxycodone and transdermal fentanyl patch. ${ }^{5}$ Patients aged $<40$ years and taking daily dosages $>120 \mathrm{mg}$ oral morphine equivalent (corresponding to fentanyl TTS $50 \mu \mathrm{g}$ /hour) showed more often breakthrough pain. ${ }^{5}$ The authors calculated that pain control with fentanyl TTS lasted for 62.9 hours on average. ${ }^{5}$ However, the authors performed a self-reported survey over the last week, and thus, the results might simply mirror that younger patients who need high opioid dosages might feel breakthrough pain more easily than older patients. Furthermore, extrapolation to TTS is questionable because oral and transdermal application forms were aggregated. Finally, stoicism is more pronounced in elderly patients whose beliefs may influence their pain experience. ${ }^{7}$ Thus, we conclude that younger patients may complain more and thus obtain higher opioid dosages.

In our study, early fentanyl TTS replacement was also associated with higher rescue dosages. However, over the 2 days of TTS application, the mean dosages of rescue medication were similar between the first 0-24 hours and the next 24-48 hours, indicating that breakthrough pain was overcome with the appropriate method and according to the best practice.

The analysis of consecutive applications from the first to the fourth TTS showed that mean dosages of the fentanyl TTS increased from one application to the next, independently of the replacement time (after 48 hours or other time) and refuted our hypothesis. Increasing the dosage of the fentanyl TTS at the end of the application interval ( 72 hours or 48 hours) seemed to be the method of choice to obtain pain control and fits to best practice.

Several studies mentioned a substantial number of cancer patients who replaced fentanyl TTS every second day because of pain aggravation before the next application. ${ }^{5,6}$ One reason for a reduction in the dosing interval is rooted in theory, ie, it is one option for obtaining higher plasma concentrations with the same drug and the same route of administration; the second option is increasing the dosage ${ }^{8}$ However, if this practice is valid for oral modified-release opioid formulations, it might be questionable for transdermal systems that were developed to deliver a constant amount of drug over several days. Moreover, transdermal systems contain substantial amounts of residual fentanyl after the usual 3 days of dosing interval. Nevertheless, it appears that some patients might not have adequate serum concentration of fentanyl over the 3 days of application, and thus, they manifest an increase in pain episodes at the second day after application of a TTS, equivalent to an end-of-dosage failure. ${ }^{9}$ We identified in the literature potential factors responsible for transient flares of severe pain, ${ }^{10}$ ie, end-of-dose failure, liver function, and the use of cytochrome P450-3A4 inducers, as the most significant factors influencing serum fentanyl concentration. ${ }^{11}$ Gene polymorphisms and demographic and disease pathology factors explain only a very small portion of variation in fentanyl concentrations. ${ }^{12}$ In our study, rationale reasons for an early replacement of fentanyl TTS could not be deduced from the patients' clinical data (no impaired liver function, no interaction with CYP inducers). In this context, some authors conclude that additional variables may explain the large interpatient variability in serum fentanyl concentrations. ${ }^{12,13}$ Hepatic clearance (ie, liver blood flow) and transdermal absorption (ie, skin temperature, attachment, sweat, loss of subcutaneous fat) are likely factors to explain the variability in serum fentanyl up to 200 -fold, even for the same dosage. ${ }^{12}$

In our survey, poor adhesion to the skin and end-of-dosage pain were the two most often answers given by professionals involved in the care of cancer patients when asked about 
the reasons for early replacement of fentanyl TTS. Good adhesion of a transdermal patch to the skin is essential for maximum efficacy, and adhesion failure hampers proper dosing. ${ }^{14}$ Consequently, and in the absence of any other rationale reason, skin adhesion problems may point practical reasons to explain an early replacement of fentanyl TTS and mimic end-of-dosage failure. This relationship needs to be confirmed in a further study.

\section{Strengths and limitations}

This study has several strengths. First, patients received a wide range of transdermal fentanyl dosages, reflecting realworld practice in pain cancer treatment. Second, quantitative data were backed up with qualitative data obtained from the health care professionals currently caring for cancer patients. Thus, this mixed method approach was able to add further facets in exploring the reasons for early transdermal patch replacement. Finally, our findings represent a real-life context and add to the current knowledge base on the very real perception for need to change dose in patients.

This study has some limitations. First, the conversion of the rescue medication into oral morphine equivalent was calculated with the equipotency references available from Swiss Hospitals. Other sources might propose other references, which might influence the final values. However, the most often used rescue medication in this study was morphine whose factor 100 is universal. Thus, the potential variations in the calculated rescue medications can be considered as marginal. Second, the rescue medication was extracted from the records and summed over 24 hours from 12 am to 11:59 pm. Because most of the fentanyl TTS were administered at $2 \mathrm{pm}$, a shift is thinkable within the amount of rescue medication calculated over each 24 hours. However, this difference concerns only the first 14 hours and merges into the next interval. As a consequence, the overall values might be marginally influenced. Third, the electronic confirmation of the administrations contained some errors. Small typing errors were corrected when the identification of the product was unequivocal (eg, one fentanyl TTS $50 \mu \mathrm{g} /$ hour was recorded as 50 fentanyl TTSs) or when the intention was unequivocal (eg, the stop time was entered as an administration time). From the total 3,122 fentanyl TTS administered to 739 patients, $27.9 \%$ were excluded because of missing data from a major field, mostly the strength. This might be due to time constraint on the wards or different interpretation of the entry fields in the database. Nevertheless, the final analysis of 2,250 fentanyl TTS allows strong results. Fourth, pain assessment was not taken into consideration. Thorough pain assessment and reassessment guide the monitoring and the dosing of the administered pain medication and are performed routinely at St Clara hospital. However, our aims were not to correlate the administered pain medication according to patients' pain scores, and thus, we focused more on data sets related to our question of interest. Finally, we did not scientifically determine the reasons for early change, such as comparison of residual content of patches or sequential blood levels.

\section{Conclusion}

In summary in our study, control of severe cancer-induced pain was obtained by increasing dosages of fentanyl TTS over consecutive applications and was accompanied by administration of constant doses of breakthrough medication, irrespective of replacement time-according to best practice. Thus, flares of pain after 48 hours seem to be the reason for early replacements of fentanyl TTS. However, in the absence of any rationale reason (physiological, pharmacological, or environmental), skin adhesion problems after 2 days may hamper delivery of the substance into the skin and thus induce subtherapeutic levels and breakthrough pain. This practical hypothesis for an early replacement of fentanyl TTS needs to be confirmed in further studies.

\section{Acknowledgment}

The Pharmaceutical Care Research Group funded this study.

\section{Author contributions}

IA, KEH, and EB made substantial contributions to conception and design. Acquisition of the data was completed by SS. All authors contributed toward data analysis, drafting and critically revising the paper and agree to be accountable for all aspects of the work.

\section{Disclosure}

The authors report no conflicts of interest in this work.

\section{References}

1. Lane ME. The transdermal delivery of fentanyl. Eur J Pharm Biopharm. 2013;84(3):449-455.

2. Adams D, Gunyea I, Bhakta B, et al. Retrospective assessment of frequency of dosing of sustained release opiate preparations in chronic pain patients [abtract of the 18th annual AAPM meeting]. Pain Med. 2002;3(2):185-185.

3. Grond S, Radbruch L, Lehmann KA. Clinical pharmacokinetics of transdermal opioids: focus on transdermal fentanyl. Clin Pharmacokinet. 2000;38(1):59-89.

4. American Pain Society. Principals of Analgesic Use in the Treatments of Acute Pain and Cancer Pain. 6th ed. Chicago: American Pain Society; 2008. 
5. Kim D-Y, Song H-S, Ahn J-S, et al. The dosing frequency of sustainedrelease opioids and the prevalence of end-of-dose failure in cancer pain control: a Korean multicenter study. Support Care Cancer. 2011; 19(2):297-301.

6. Radbruch L, Elsner F. Clinical experience with transdermal fentanyl for the treatment of cancer pain in Germany. Keio J Med. 2004;53(1): 23-29.

7. Abdulla A, Adams N, Bone M, et al. Guidance on the management of pain in older people. Age Ageing. 2013;42(suppl 1):i1-i57.

8. Gourlay GK. Treatment of cancer pain with transdermal fentanyl. Lancet Oncol. 2001;2(3):165-172.

9. Koike K, Terui T, Nagasako T, et al. A new once-a-day fentanyl citrate patch (Fentos ${ }^{\circledR}$ Tape) could be a new treatment option in patients with end-of-dose failure using a 72-h transdermal fentanyl matrix patch. Support Care Cancer. 2015;24(3):1053-1059.
10. Portenoy RK, Hagen NA. Breakthrough pain: definition, prevalence and characteristics. Pain. 1990;41(3):273-281.

11. Kokubun H, Ebinuma K, Matoba M, Takayanagi R, Yamada Y, Yago K. Population pharmacokinetics of transdermal fentanyl in patients with cancer-related pain. J Pain Palliat Care Pharmacother. 2012;26(2):98-104.

12. Barratt DT, Bandak B, Klepstad P, et al. Genetic, pathological and physiological determinants of transdermal fentanyl pharmacokinetics in 620 cancer patients of the EPOS study. Pharmacogenet Genomics. 2014; 24(4):185-194.

13. Solassol I, Bressolle F, Caumette L, et al. Inter- and intraindividual variabilities in pharmacokinetics of fentanyl after repeated 72-hour transdermal applications in cancer pain patients. Ther Drug Monit. 2005;27(4):491-498.

14. Lampert A, Seiberth J, Haefeli WE, Seidling HM. A systematic review of medication administration errors with transdermal patches. Expert Opin Drug Saf. 2014;13(8):1101-1114.
Journal of Pain Research

\section{Publish your work in this journal}

The Journal of Pain Research is an international, peer reviewed, open access, online journal that welcomes laboratory and clinical findings in the fields of pain research and the prevention and management of pain. Original research, reviews, symposium reports, hypothesis formation and commentaries are all considered for publication.

\section{Dovepress}

The manuscript management system is completely online and includes a very quick and fair peer-review system, which is all easy to use. Visit http://www.dovepress.com/testimonials.php to read real quotes from published authors. 